\title{
Low bone mineral density in COPD patients related to worse lung function, low weight and decreased fat-free mass
}

\author{
A. Vrieze • M. H. G. de Greef • P. J. Wýkstra • \\ J. B. Wempe
}

Received: 14 September 2006 / Accepted: 6 February 2007 / Published online: 9 March 2007

(C) International Osteoporosis Foundation and National Osteoporosis Foundation 2007

\begin{abstract}
Summary Low bone mineral density is frequently seen in COPD patients. Advanced COPD, low BMI and muscle depletion are risk factors for developing low bone mineral density (BMD). Low bone mineral density is seen in $75 \%$ of the GOLD stage IV patients.

Introduction We set out to investigate the prevalence of low bone mineral density (BMD) in chronic obstructive pulmonary disease (COPD) as well as the predictors of abnormal bone mineral density.

Methods A cross-sectional design was used to evaluate 115 subjects with COPD (GOLD stages II-IV). Bone mineral density (BMD) was measured using an ultrasound densitometer. The forced expiratory volume in $1 \mathrm{~s}\left(\mathrm{FEV}_{1}\right)$ was assessed and fat-free mass was measured using bioelectrical impedance analysis. Chi-square tests and logistic regression were used for analysis.

Results The prevalence of a T-score $<-1.0 \mathrm{SD}$ and $>-2.5$ SD was $28.6 \%$ in GOLD stage II, $40.3 \%$ in GOLD stage III and $57.1 \%$ in GOLD stage IV. The prevalence of a T-score $\leq-2.5 \mathrm{SD}$ was $0 \%$ in GOLD stage II, $9.6 \%$ in GOLD stage
\end{abstract}

A. Vrieze $\cdot$ M. H. G. de Greef

Institute of Human Movement Sciences, University of Groningen,

Groningen, The Netherlands

A. Vrieze · J. B. Wempe

Center for Rehabilitation, University Medical Center Groningen,

Groningen, The Netherlands

\section{P. J. Wýkstra · J. B. Wempe $(\bowtie)$}

Department of Pulmonary Diseases,

University Medical Center Groningen,

P.O. Box 30001, 9700 RB Groningen, The Netherlands

e-mail: j.b.wempe@int.umcg.nl

P. J. Wýkstra

e-mail: p.j.wykstra@int.umcg.nl
III and $17.9 \%$ in GOLD stage IV. In a logistic model FFM, BMI and $\mathrm{FEV}_{1}$ were significant predictors of abnormal bone mineral density. Patients in GOLD stage IV have a 7.6 times greater risk of abnormal bone mineral density than patients in GOLD stage II.

Conclusions Low bone mineral density is frequently present in COPD patients. Low FFM, BMI and $\mathrm{FEV}_{1}$ are risk factors for developing a low T-score. A low FFM or BMI in GOLD stage IV strongly suggests loss of BMD and warrants further examination.

Keywords Chronic obstructive pulmonary disease . Fat-free mass - Low bone mineral density $\cdot$ Lung function

\section{Introduction}

Patients with chronic obstructive pulmonary disease (COPD) have a high risk of developing osteopenia and osteoporosis $[1,2]$. Osteopenia (T-score between -1.0 and -2.5 ) and osteoporosis (T-score below -2.5 ) are conditions characterized by a decrease in bone mass and density, causing bones to become fragile. It is still not entirely clear which mechanisms cause this problem [3]. Known risk factors for developing osteoporosis in COPD are aging, female sex, impaired nutritional status, low exercise levels, corticosteroids and tobacco smoking [4-6].

Nowadays, COPD is considered as a pulmonary disease with many systemic manifestations. Patients with COPD may have, in addition to a change in BMD, lowered fat-free mass (FFM), muscle strength, endurance capacity, BMI and heart failure. One explanation for these systemic effects is the increase in systemic inflammation in COPD, which is suggested by high levels of CRP, oxidative stress and other (pro-) inflammatory mediators. The most important factor 
in this inflammatory process is tobacco smoke. Tobacco smoke directly induces systemic damage, for instance, by lowering the $\mathrm{pH}$ of bone tissue, resulting in absorption of bone salts [7], but also has indirect systemic effects by inducing an inflammatory response in the lungs with production of systemic inflammatory mediators with effects on bone and other organs [8]. Another explanation for the low BMD in COPD is the low level of physical activity of subjects with COPD. Patients with COPD experience dyspnea during exertion, which may lead to inactivity with negative effects on BMD [9].

In this study we examined the prevalence of a low bone mineral density in COPD. Furthermore, the relationship of BMD with lung function and two parameters which are considered as parameters of systemic inflammation, namely BMI and FFM, were studied.

\section{Methods}

\section{Study design}

A cross-sectional design was used to evaluate subsequent subjects with COPD (GOLD stages II-IV) who were referred for pulmonary rehabilitation to the Rehabilitation Center of the University Medical Center Groningen. Subjects were included if they had COPD GOLD stages II-IV, were 40 years of age or older, and were in clinically stable condition. Patients aged 80 and older were excluded from this study.

\section{Measurements}

All subjects underwent a standardized measurement program before the rehabilitation period in which lung function, height and weight were taken and BMI was calculated. The bioelectrical impedance, for calculation of fat-free mass, was not measured in the first group of patients, but only in the last 58 patients.

Bone mineral density measurement

We used quantitative ultrasound (QUS) for the assessment of skeletal status. QUS is a peripheral bone densitometry technique that is a useful screening technique, as it is believed to provide not only an estimate of regional bone mineral density (BMD), but also to evaluate bone quality [10] and predict fractures risk [11]. Dual-energy X-ray absorptiometry (DEXA) method is considered to be the gold standard to measure bone mineral density (BMD), [12, 13], but research has shown that ultrasound densitometry is a valid and reliable alternative for the DEXA method [1416]. QUS results correlate well with BMD measured by DEXA at the heel [17]. Although calcaneal QUS devices cannot formally diagnose osteoporosis, as they do not actually measure BMD, they perform similarly to DEXA when discriminating for patients with osteoporotic fracture [18]. QUS has the advantage of not using ionizing radiation. It is less expensive, more time-efficient and easier to use than densitometry techniques.

In this study evaluation of skeletal status was based on calcaneal QUS measurements, carried out by the SAHARA Clinical Bone Sonometer (Hologic, Bedford, MA, USA). Broadband ultrasound attenuation (BUA) and speed of sound (SOS) were measured at a fixed region in the calcaneus of the dominant foot. BUA and SOS were then combined into a single parameter, the quantitative ultrasound index (QUI). An estimate of the heel BMD (eBMD) was also derived. The estimated heel BMD is inferred from a linear combination of BUA and SOS, and is not an actual measurement of calcaneal BMD. T-scores for all subjects were calculated on the basis of eBMD. T-score is defined as the difference between the patient's results and the mean results obtained in a young population, expressed in units of standard deviations (SD). Based on manufacturerderived T-scores for eBMD of the dominant heel, subjects were classified into categories, i.e., greater than or equal to $-12 \mathrm{SD}$, between -1 and $-2.5 \mathrm{SD}$, and lower than or equal to $-2.5 \mathrm{SD}$.

\section{Lung function}

The forced expiratory volume in $1 \mathrm{~s}\left(\mathrm{FEV}_{1}\right)$ was measured with the Masterscreen PFT (Jaeger, Hoechberg, Germany). This computerized spirometer is reliable ( $\mathrm{r}=0.84$ to 0.96 ) and valid, according to the American Thoracic Society standards [19]. The $\mathrm{FEV}_{1} \%$ pred was used to determine the level of COPD using the global initiative of obstructive lung disease (GOLD) [20]. The GOLD classifies patients into different stages based on $\mathrm{FEV}_{1} \%$ pred. Table 1 shows the classification of COPD in GOLD stages.

Fat-free mass / fat-free mass index

Fat-free mass (FFM) and fat-free mass index (FFMI) were measured using bioelectrical impedance analysis (Biostat

Table 1 Classification of COPD in GOLD stages

\section{Stage}

\begin{tabular}{ll} 
I: At risk & $\mathrm{FEV}_{1} \geq 80 \%$ predicted \\
II: Mild COPD & $50 \% \leq \mathrm{FEV}_{1}<80 \%$ predicted \\
III: Moderate COPD & $30 \% \leq \mathrm{FEV}_{1}<50 \%$ predicted \\
IV: Severe COPD & $\begin{array}{l}\mathrm{FEV}_{1}<30 \% \text { predicted, or the presence of } \\
\text { respiratory failure* }\end{array}$ \\
\hline
\end{tabular}

*Respiratory failure: $\mathrm{PaO} 2<8.0 \mathrm{kPa}(60 \mathrm{~mm} \mathrm{Hg})$ with or without $\mathrm{PaCO} 2>6.7 \mathrm{kPa}(50 \mathrm{~mm} \mathrm{Hg})$ while breathing air at sea level. 
Table 2 Baseline

characteristics
BMI, body mass index; FFMI, fat-free mass index, $\mathrm{FEV}_{1}$, forced expiratory volume in one second

\begin{tabular}{lllll}
\hline & Total group & GOLD stage II & GOLD stage III & GOLD stage IV \\
\hline Male/female (N) & $62 / 53$ & $18 / 17$ & $26 / 26$ & $18 / 10$ \\
Age (y) & $60.0 \pm 10.8$ & $59.3 \pm 12.4$ & $61.6 \pm 10.2$ & $57.9 \pm 9.6$ \\
Body composition & & & & \\
BMI $\left(\mathrm{kg} \cdot \mathrm{m}^{-2}\right)$ & $26.4 \pm 6.4$ & $28.9 \pm 6.4$ & $26.8 \pm 6.3$ & $22.6 \pm 5.0$ \\
FFMI $\left(\mathrm{kg} \cdot \mathrm{m}^{-2}\right)$ & $17.3 \pm 3.4$ & $18.6 \pm 3.5$ & $17.4 \pm 3.6$ & $16.3 \pm 2.9$ \\
BMD & & & & \\
T-score & $-0.90 \pm 1.18$ & $-0.45 \pm 0.92$ & $-0.89 \pm 1.15$ & $-1.5 \pm 1.30$ \\
Pulmonary function & & & & \\
FEV $_{1}$ (L) & $1.26 \pm 0.59$ & $1.90 \pm 0.53$ & $1.12 \pm 0.32$ & $0.74 \pm 0.22$ \\
FEV $_{1}(\%$ predicted) & $43.4 \pm 16.9$ & $64.7 \pm 8.9$ & $39.8 \pm 6.0$ & $23.6 \pm 4.6$ \\
\hline
\end{tabular}

1500, Bodystat Ltd., Douglas, Isle of Man, UK). Low fatfree mass was defined as $15 \mathrm{~kg} / \mathrm{m}^{2}$ or less for women and $16 \mathrm{~kg} / \mathrm{m}^{2}$ or less for men [21]. Height and weight were determined barefoot and body mass index (BMI=weight/ height ${ }^{2}$ ) was calculated. Low BMI was defined as $18.5 \mathrm{~kg} / \mathrm{m}^{2}$ or less, based on WHO criteria.

\section{Data analysis}

The Statistical Package for Social Sciences (SPSS), version 12.0 , was used to analyze the data. Results are presented as means \pm standard deviation. Differences in prevalence of Tscores for the different GOLD stages, low/normal FFMI and low/normal BMI were tested with the Chi-square test. Logistic regression analysis was performed to determine explaining variables of abnormal bone mineral density in patients with COPD. Age, gender, $\mathrm{FEV}_{1}$, FFMI and BMI were included as the covariates. We computed the odds ratio (OR) and its $95 \%$ confidence intervals $(\mathrm{CI})$. All pvalues $<0.05$ were considered statistically significant.

\section{Results}

Participant characteristics

We evaluated 115 subjects with COPD (GOLD stages IIIV). BMI was measured in 114 patients, fat-free mass index (FFMI) in 58 patients. The characteristics of the patients are shown in Table 2.

Figures 1, 2 and 3 shows the bone mineral density for the different GOLD stages, low/normal BMI and low/ normal FFM.

Figure 1 shows that the prevalence of a low BMD increases with a higher GOLD stage. The prevalence of Tscores between -1 and -2.5 was $28.6 \%$ in GOLD stage II, $40.3 \%$ in GOLD stage III and $57.1 \%$ in GOLD stage IV. Prevalence of T-scores lower than or equal to -2.5 was $0 \%$ in GOLD stage II, $9.6 \%$ in GOLD stage III and $17.90 \%$ in
GOLD stage IV. There is a significant difference in bone mineral density for the different GOLD stages $\left(\chi^{2}=15.6\right.$, $\mathrm{df}=4, \mathrm{p}=0.004)$.

Figure 2 shows the prevalence of a low BMD for underweight and normal-weight patients. Of the 114 patients, 104 had a normal BMI $\left(>18.5 \mathrm{~kg} / \mathrm{m}^{2}\right)$ and 10 had a low BMI $\left(\leq 18.5 \mathrm{~kg} / \mathrm{m}^{2}\right)$. Of the underweight patients, $20 \%$ had a normal BMD, $60 \%$ had a T-score between -1 and -2.5 and $20 \%$ had a T-score lower than or equal to -2.5 . Of the patients with normal weight, $52.9 \%$ had normal BMD, $39.4 \%$ a T-score between -1 and -2.5 and $7.7 \%$ had a $\mathrm{T}$-score lower than or equal to -2.5 . Although it seems that the risk of a low bone mineral density increased with low BMI, the difference in bone mineral density for BMI is not significant $\left(\chi^{2}=4.9, \mathrm{df}=2\right.$, $\mathrm{p}>0.05$ ).

Figure 3 shows the prevalence of low BMD for patients with low and normal FFM. Of the 58 patients whose FFM was measured, 20 patients had low FFM and 38 had normal FFM. Of the 20 patients with low FFM, 20\% had normal BMD, $60 \%$ a T-score between -1 and -2.5 and $20 \%$ had a T-score lower than or equal to -2.5 . Of the 38 patients with normal FFM, 55.3\% had normal BMD, 34.2\% a T-score between -1 and -2.5 and $10.5 \%$ had a T-score lower than or equal to -2.5 . There is a significant difference in bone

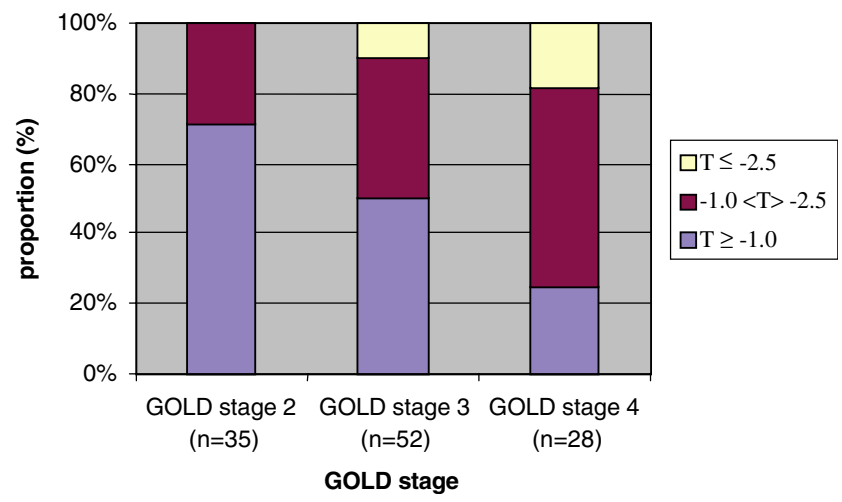

Fig. 1 Bone mineral density for the different GOLD stages 


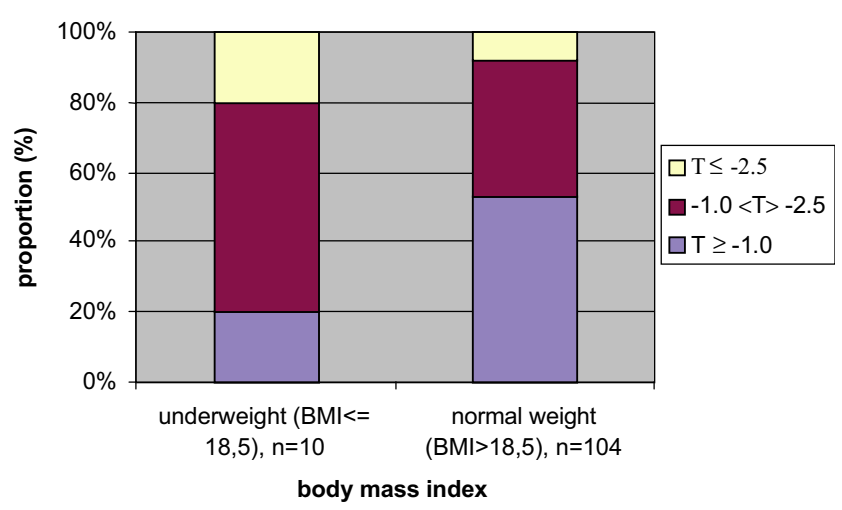

Fig. 2 Bone mineral density for underweight and normal-weight patients

mineral density for FFM $\left(\chi^{2}=6.7, d f=2, p=0.036\right)$. FFM decreased with increasing severity of COPD and the risk of low BMD increased with low FFM.

Predictors of abnormal bone mineral density

We also examined the predictors of abnormal bone mineral density (T-score $<-1.0$ ) with logistic regression. In our patients with COPD GOLD stages II-IV, FFM and BMI were the most dominant predictors of low BMD. $\mathrm{FEV}_{1}$ was found to be another relevant predictor of low BMD. Gender and age were not significant predictors. Tables 3 and 4 shows the results of logistic regression.

To determine whether the prevalence of abnormal bone mineral density was higher in patients with GOLD IV than in GOLD II, logistic regression analyses were performed. The results are shown in Table 4. When the results were adjusted for age and gender, the risk of abnormal bone mineral density was 7.6 times greater for patients with GOLD IV than for patients with GOLD II (OR 7.6, 95\% CI 2.4 to 24.3 ). The risk of abnormal bone mineral density was 4.9 times greater for patients with low FFM than for patients with normal FFM. (OR 4.9, 95\% CI 1.3 to 17.8 ).

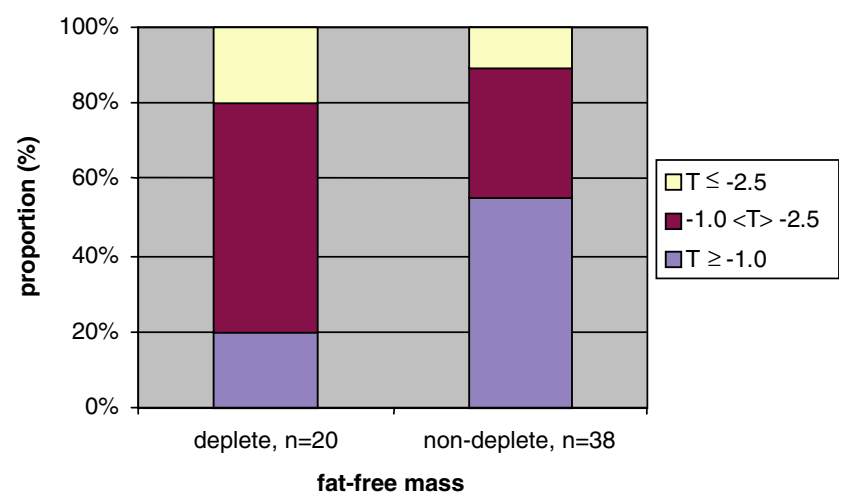

Fig. 3 Bone mineral density for patients with low FFM (depleted) and normal FFM (non-depleted)
Table 3 Logistic regression model with covariates: age, gender, $\mathrm{FEV}_{1}$ and FFMI

\begin{tabular}{llll}
\hline & $\mathrm{B}(\mathrm{SE})$ & Exp (B) & $95 \% \mathrm{CI}$ \\
\hline Constant & $9.010(3.144)$ & & \\
Age & $-0.044(0.034)$ & 0.957 & $0.896-1.02$ \\
Gender & $-0.954(0.682)$ & 0.385 & $0.101-1.47$ \\
FFMI, kg/m & $-0.235(0.107)^{*}$ & 0.791 & $0.642-.975$ \\
FEV $_{1}, 1$ & $-1.290(0.620)^{*}$ & 0.275 & $0.082-.927$ \\
\hline
\end{tabular}

Dependent is abnormal bone mineral density (T-score $<-1 \mathrm{SD}$ ) $\mathrm{R}^{2}=0.20$ (Cox \& Snell), 0.27(Nagelkerke) $* \mathrm{p}<0.05$

The BMI also influence the risk of abnormal bone mineral denisty, the risk is 4.7 times higher for patients with low BMI than for patients with normal BMI (OR 4.7, $95 \% \mathrm{CI}$ .96 to 23.7$)$.

\section{Discussion}

The present study shows a high prevalence of low BMD in COPD. This prevalence rises from $28.6 \%$ in GOLD stage II to $75.0 \%$ in GOLD stage IV. Patients in GOLD IV have a 7.6 times greater risk of low BMD than patients with GOLD II. In addition, a low FFM and, to a slightly less extent, low BMI both increase the risk of low BMD. Patients with low FFM have a 4.9 times greater risk of low BMD than patients with a normal FFM. Low BMI increases the risk of low BMD with 4.7 times. Logistic regression showed that FFM, BMI and $\mathrm{FEV}_{1}$ are significant predictors of abnormal bone mineral density (Table 5).

Our results are in line with previous studies. It has been demonstrated that patients with advanced COPD are at risk for having low fat-free mass [22]. Bolton et al. showed that $32 \%$ of the 81 COPD patients with an FEV1 of $16-105 \%$

Table 4 Logistic regression model with covariates: age, gender, $\mathrm{FEV}_{1}$ and BMI

\begin{tabular}{llll}
\hline & $\mathrm{B}(\mathrm{SE})$ & $\operatorname{Exp}(\mathrm{B})$ & $95 \% \mathrm{CI}$ \\
\hline Constant & $3.294(1.628)$ & & \\
Age & $0.000(0.019)$ & 1.000 & $0.964-1.039$ \\
Gender & $0.414(0.434)$ & 0.661 & $0.282-1.549$ \\
BMI, $\mathrm{kg} / \mathrm{m}^{2}$ & $-0.081(0.035)^{*}$ & 0.922 & $0.861-0.988$ \\
$\mathrm{FEV}_{1}, 1$ & $-0.800(0.398)^{*}$ & 0.449 & $0.206-0.980$ \\
\hline
\end{tabular}

Dependent is abnormal bone mineral density (T-score $<-1 \mathrm{SD}$ ) $\mathrm{R}^{2}=0.12$ (Cox \& Snell), 0.16 (Nagelkerke) $* \mathrm{p}<0.05$ 
Table 5 Three different logistic regression models for abnormal BMD in COPD patients

\begin{tabular}{lll}
\hline & $\mathrm{N}$ & Adjusted OR $(95 \% \mathrm{CI})$ \\
\hline GOLD II & 35 & 1.0 \\
GOLD III & 52 & $2.4(0.97$ to 6.1$) *$ \\
GOLD IV & 28 & $7.6(2.4$ to 24.3$) *$ \\
Normal FFM & 38 & 1.0 \\
Low FFM & 20 & $4.9(1.3$ to 17.8$) *$ \\
Normal BMI & 104 & 1.0 \\
Low BMI & 10 & $4.7(0.96$ to 23.7$) *$ \\
\hline
\end{tabular}

Results are adjusted for age and gender $* \mathrm{p}<0.05$

pred. had osteoporosis, assessed by DEXA [23]. They did not report a link between BMD and lung function, but clearly showed that a low FFMI or BMI was associated with increased protein catabolism and low BMD. Very recently, Jørgensen showed that $68 \%$ of 62 COPD patients with a mean FEV1 of $33 \%$ pred. had osteopenia or osteoporosis, assessed by DEXA or spinal X-ray [2]. Our research adds to these studies that both low lung function and fat-free mass are associated with bone loss.

There may be several explanatory mechanisms for our results. The first is the presence of systemic inflammation. Among others, Gan et al. [24] found that reduced lung function is associated with increased levels of systemic inflammatory markers. In addition, systemic inflammation is being recognized as a risk factor for osteoporosis, cachexia and weight loss [24].

The second mechanism of reduced bone mass in COPD is a low level of physical activity. Patients with COPD experience dyspnea during exertion, which may lead to a vicious circle of inactivity, deconditioning and increased dyspnea on exertion. Recently, Pitta et al. [9] showed that most patients with COPD spend less time walking and standing and more time sitting and lying in daily life when compared with sedentary healthy elderly subjects. It would be compelling to study the effect on bone density of interventions aiming at increasing daily physical activity. In fact, it has already been shown that patients with COPD present an improvement in bone mineral density after lung volume reduction surgery, which has been ascribed to an increase in physical activity [25].

The third mechanism is the corticosteroid-induced loss of bone mass. In our study, the majority of patients were on inhaled corticosteroids, especially in GOLD stage IV. Many more patients with severe COPD had intermittent courses of oral corticosteroids, but as we had no sufficient details on previous corticosteroid medication, we were not able to demonstrate a statistical effect of (inhaled) corticosteroid dose on BMD.
Limitations of the study

We used the Sahara clinical bone sonometer to assess skeletal status. Dual energy X-ray absorptiometry (DEXA) is the most widely used and preferred quantitative technique for the assessment of skeletal status. Bone mineral density measured by DEXA is the best predictor of fracture risk and is currently considered the gold standard to diagnose osteoporosis. The QUS can be used as a screening technique, as it is believed to give an estimate of the BMD and predict fracture risk [11]. Frost et al. showed that DEXA and QUS were able to a similar extent to discriminate the risk of fractures in postmenopausal women [26].

Calcaneal QUS devices cannot formally diagnose osteoporosis, though, because they do not actually measure BMD. The QUS test relies on an indirect measurement of the anatomy (heel) to quantify the amount of bone loss in other more direct areas of the body. The QUS can be utilized as a screening test for osteoporosis, but up to $10 \%$ of patients will get a "false normal" test result despite having clinically significant osteoporosis [27]. QUS may have a role in defining the COPD patients who should have a DEXA scan.

Corticosteroid treatment diminishes bone mineral density, especially in the vertebrae. We measured the bone mineral density at the calcaneus. A scan of the calcaneus may thus give an underestimation of the bone mineral density in COPD patients using corticosteroids, suggesting an even higher prevalence of low BMD.

\section{Clinical implications}

The results of our study show that patients with advanced COPD (GOLD stage IV) are a risk group for developing low BMD. In GOLD stage IV $75 \%$ of the patients have a low BMD. Because of the predicted worldwide increase in COPD with subsequent bone problems such as fractures, there should be an identification of COPD patients with a high risk of osteoporosis. There recommend extra attention for patients with advanced COPD, especially if they also have low FFMI and/or low BMI.

In summary, this study shows that a low BMD is frequently present in COPD. Advanced COPD, low BMI and low FFM are risk factors for the presence of low BMD. Abnormal bone mineral density is seen in $75 \%$ of the GOLD stage IV patients. FFM, BMI and $\mathrm{FEV}_{1}$ are the most important predictors of low BMD in COPD patients. Future studies are needed to determine the best way to identify high-risk patients and whether early treatments such as lifestyle interventions or medication are able to reverse the osteoporotic process. 


\section{References}

1. Papaioannou A, Parkinson W, Ferko N, Probyn L, Ioannidis G, Jurriaans E et al (2003) Prevalence of vertebral fractures among patients with chronic obstructive pulmonary disease in Canada. Osteoporos Int 14(11):913-917

2. Jorgensen NR, Schwarz P, Holme I, Henriksen BM, Petersen LJ, Backer V (2006) The prevalence of osteoporosis in patients with chronic obstructive pulmonary disease-A cross sectional study. Respir Med

3. Katsura H, Kida K (2002) A comparison of bone mineral density in elderly female patients with COPD and bronchial asthma. Chest 122(6):1949-1955

4. Biskobing DM (2002) COPD and osteoporosis. Chest 121 (2):609-620

5. Cummings SR, Nevitt MC, Browner WS, Stone K, Fox KM, Ensrud KE et al (1995) Risk factors for hip fracture in white women. Study of Osteoporotic Fractures Research Group. N Engl J Med 332(12):767-773

6. Law MR, Hackshaw AK (1997) A meta-analysis of cigarette smoking, bone mineral density and risk of hip fracture: recognition of a major effect. BMJ 315(7112):841-846

7. Daniell HW (1972) Osteoporosis and smoking. JAMA 221(5):509

8. Agusti AG, Noguera A, Sauleda J, Sala E, Pons J, Busquets X (2003) Systemic effects of chronic obstructive pulmonary disease. Eur Respir J 21(2):347-360

9. Pitta F, Troosters T, Spruit MA, Probst VS, Decramer M, Gosselink R (2005) Characteristics of physical activities in daily life in chronic obstructive pulmonary disease. Am J Respir Crit Care Med 171(9):972-977

10. Gluer CC (1997) Quantitative ultrasound techniques for the assessment of osteoporosis: expert agreement on current status. The International Quantitative Ultrasound Consensus Group. J Bone Miner Res 12(8):1280-1288

11. Hans D, Dargent-Molina P, Schott AM, Sebert JL, Cormier C, Kotzki PO et al (1996) Ultrasonographic heel measurements to predict hip fracture in elderly women: the EPIDOS prospective study. Lancet 348(9026):511-514

12. Mautalen CA (2001) Osteoporosis in Argentina. Clin Calcium 11 (4):496-498

13. Sadat-Ali M, Al Habdan IM, Al Mulhim FA, El Hassan AY (2004) Bone mineral density among postmenopausal Saudi women. Saudi Med J 25(11):1623-1625

14. Nogata F, Matsui K, Kagechika K, Sueyoshi Y, Tomita K (1999) Estimation of in vivo bone mineral density (BMD) and shape characterization for diagnosing osteoporosis by ultrasonic inspection. J Biomech Eng 121(3):298-303
15. Hartman C, Shamir R, Eshach-Adiv O, Iosilevsky G, Brik R (2004) Assessment of osteoporosis by quantitative ultrasound versus dual energy X-ray absorptiometry in children with chronic rheumatic diseases. J Rheumatol 31(5):981-985

16. Lusenti T, Cadossi R, Franco V, Soliani F, Rustichelli R, Borgatti $P$ (1994) Ultrasound evaluation of the bone tissue of the proximal phalanges of hands in patients with suspected type I osteoporosis. Minerva Ginecol 46(7-8):423-428

17. Langton CM, Njeh CF, Hodgskinson R, Currey JD (1996) Prediction of mechanical properties of the human calcaneus by broadband ultrasonic attenuation. Bone 18(6):495-503

18. Magkos F, Manios Y, Babaroutsi E, Sidossis LS (2005) Quantitative ultrasound calcaneus measurements: normative data for the Greek population. Osteoporos Int 16(3):280-288

19. Gardner RM, Hankinson JL (1988) Standardization of spirometry1987 ATS update (American Thoracic Society). J Occup Med 30 (3):272-273

20. Pauwels RA, Buist AS, Calverley PM, Jenkins CR, Hurd SS (2001) Global strategy for the diagnosis, management, and prevention of chronic obstructive pulmonary disease. NHLBI/WHO Global Initiative for Chronic Obstructive Lung Disease (GOLD) Workshop summary. Am J Respir Crit Care Med 163(5):1256-1276

21. Vermeeren MA, Creutzberg EC, Schols AM, Postma DS, Pieters WR, Roldaan AC et al (2006) Prevalence of nutritional depletion in a large out-patient population of patients with COPD. Respir Med

22. Vestbo J, Prescott E, Almdal T, Dahl M, Nordestgaard BG, Andersen $\mathrm{T}$ et al (2006) Body mass, fat-free body mass, and prognosis in patients with chronic obstructive pulmonary disease from a random population sample: findings from the Copenhagen City Heart Study. Am J Respir Crit Care Med 173(1):79-83

23. Bolton CE, Ionescu AA, Shiels KM, Pettit RJ, Edwards PH, Stone MD et al (2004) Associated loss of fat-free mass and bone mineral density in chronic obstructive pulmonary disease. Am J Respir Crit Care Med 170(12):1286-1293

24. Gan WQ, Man SF, Senthilselvan A, Sin DD (2004) Association between chronic obstructive pulmonary disease and systemic inflammation: a systematic review and a meta-analysis. Thorax 59 (7):574-580

25. Mineo TC, Ambrogi V, Mineo D, Fabbri A, Fabbrini E, Massoud R (2005) Bone mineral density improvement after lung volume reduction surgery for severe emphysema. Chest 127(6):1960-1966

26. Frost ML, Blake GM, Fogelman I (2002) A comparison of fracture discrimination using calcaneal quantitative ultrasound and dual X-ray absorptiometry in women with a history of fracture at sites other than the spine and hip. Calcif Tissue Int 71(3):207-211

27. Moyad MA (2003) Osteoporosis: a rapid review of risk factors and screening methods. Urol Oncol 21(5):375-379 